\title{
Incidental Prostate Carcinoma Diagnosed at Radical Cystoprostatectomy for Bladder Carcinoma: A Case Report
}

\author{
Sonal Paul, Anitha Padmanabhan* and Nitin M Gadgil \\ Department of Pathology, Lokmanya Tilak Medical college and General Hospital, Sion, Mumbai, India
}

\section{ABSTRACT}

Introduction: The incidence of concomitant urothelial carcinoma of the bladder and adenocarcinoma of the prostate varies from $17 \%$ to $70 \%$ as per various literature studies.

Case report: We report the case of 82 year old male who presented with hematuria and lower urinary tract symptoms. A cystoscopy was performed which was suggestive of a $6 \times 4 \times 5 \mathrm{~cm}$ mass arising from the left anterolateral wall and dome of the bladder. A Transurethral biopsy of the bladder mass showed a high grade papillary urothelial carcinoma invading the muscularis propria. Following this, the patient underwent a radical cystoprostatectomy. Histopathological examination of the bladder mass confirmed High -grade urothelial carcinoma. The entire prostate was sampled which revealed Adenocarcinoma prostate, Gleason score 3+4=7, tumor volume $6 \mathrm{cc}$.

Conclusion: The incidence of incidentally detected prostatic adenocarcinoma in Carcinoma bladder cases is extremely variable. Complete sampling of the prostate helps in detecting these lesions.

\section{Keywords: Carcinoma Prostate, Cystoprostatectomy, Incidental, Urothelial Carcinoma Bladder}

\section{Introduction}

According to literature, the frequency of incidentally discovered carcinoma prostate in cystoprostatectomy specimens is extremely variable ranging from $4 \%$ to $60 \%$ .$^{[1,2]}$ The yield of detection of these tumors depends on the complete sampling of the prostate and the thickness of the sections taken. ${ }^{[3]}$ The incidence of clinically significant prostate adenocarcinoma varies from $5 \%$ to $48 \%$ depending upon the criteria in different studies. ${ }^{[4,5]}$ However, the prognosis depends upon the stage of the Carcinoma bladder and prostatic adenocarcinoma has no prognostic implications ${ }^{[4]}$ We report a case of clinically significant prostate adenocarcinoma detected incidentally in a radical cystoprostatectomy specimen.

\section{Case report}

We report the case of a 82 year old male who presented with hematuria for three months and lower urinary tract symptoms for one month. Patient was a chronic tobacco chewer for forty years.

All hematological investigations and biochemical investigations including Blood Urea Nitrogen and Serum creatinine were within normal limits. Serum Prostate Specific Antigen(PSA) levels were $3.4 \mathrm{ng} / \mathrm{ml}$. A Computed Tomography scan showed a $6.3 \times 4.5 \times 5 \mathrm{~cm}$ heterogeneously enhancing lesion arising from the left anterolateral wall, and dome of the bladder. Urine cytology was reported as High- Grade Urothelial Carcinoma. This was followed by a Transurethral biopsy of the bladder mass which on histopathology was reported as Muscle invasive Highgrade papillary urothelial carcinoma.

A radical cystoprostatectomy (RCP) was performed and the specimen was received in the histopathology section of our department. The specimen measured $11 \times 10 \times 6.5 \mathrm{~cm}$ with attached ureters and seminal vesicles . On opening, an exophytic friable papillary tumor measuring $7 \times 3 \times 2 \mathrm{~cm}$, completely obliterating the lumen was seen arising from the left anterolateral wall of the bladder[fig.1]. The rest of the bladder mucosa was edematous. Cut section of the tumour showed areas of hemorrhage and necrosis. Prostate measured $5 \times 4 \times 3 \mathrm{~cm}$. Cut section of the prostate was greyish white nodular. No yellow areas were seen.

Tissue sections were obtained from the primary tumor including the areas of deepest invasion, bladder wall, ureters, seminal vesicles and lymph nodes. The apex of the prostate was shaved perpendicular to the prostatic urethra. The bladder neck margin was coned from the specimen and perpendicularly sectioned. The remaining prostatic tissue was sectioned at $3 \mathrm{~mm}$ intervals along a plane perpendicular to the urethral axis and was submitted for histopathology.

On histopathology, a pT2aN0M0, High- grade papillary urothelial carcinoma (WHO 2016 Classification) [fig.2] invading the inner half of muscularis propria was seen. There was no evidence of any lymphovascular or perineural 
invasion. Also, prostate showed a T2aNxMx (WHO 2016 Classification) Acinar Adenocarcinoma involving the right lobe, with Gleason score 3+4=7 [fig. 3,4] with presence of perineural invasion. Volume of tumour was $6 \mathrm{cc}$. There was no evidence of extraprostatic extension, invasion into the bladder neck or invasion into the intraprostatic seminal vesicles. No lymphovascular invasion was seen. Six lymph nodes ranging in size from $0.5 \mathrm{~cm}$ to $0.8 \mathrm{cms}$ were dissected and all were free of tumour. There was no evidence of any

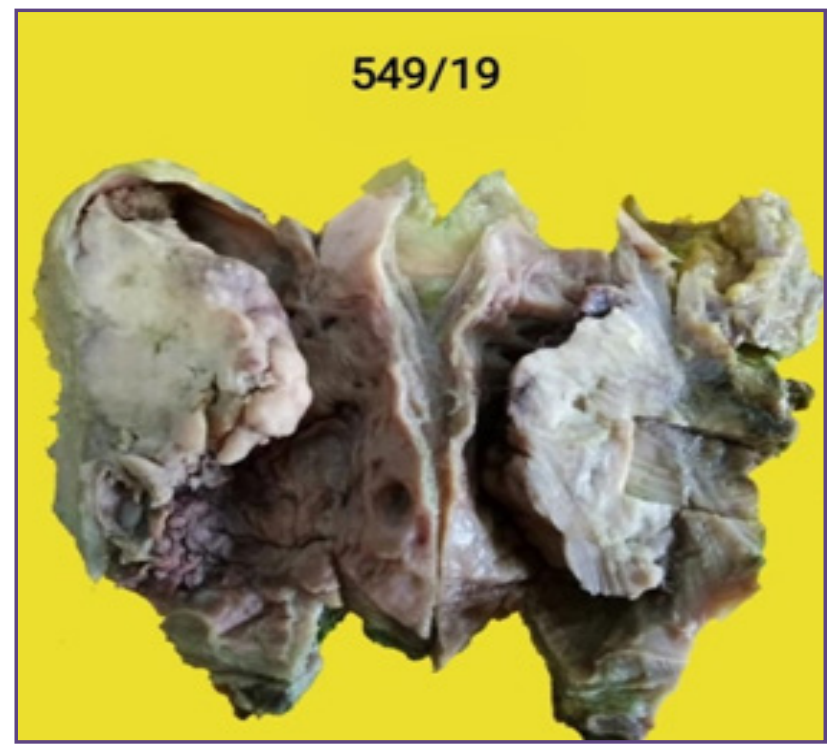

Fig. 1: Urinary bladder cut surface showing an exophytic greyish white mass arising from the lateral wall.

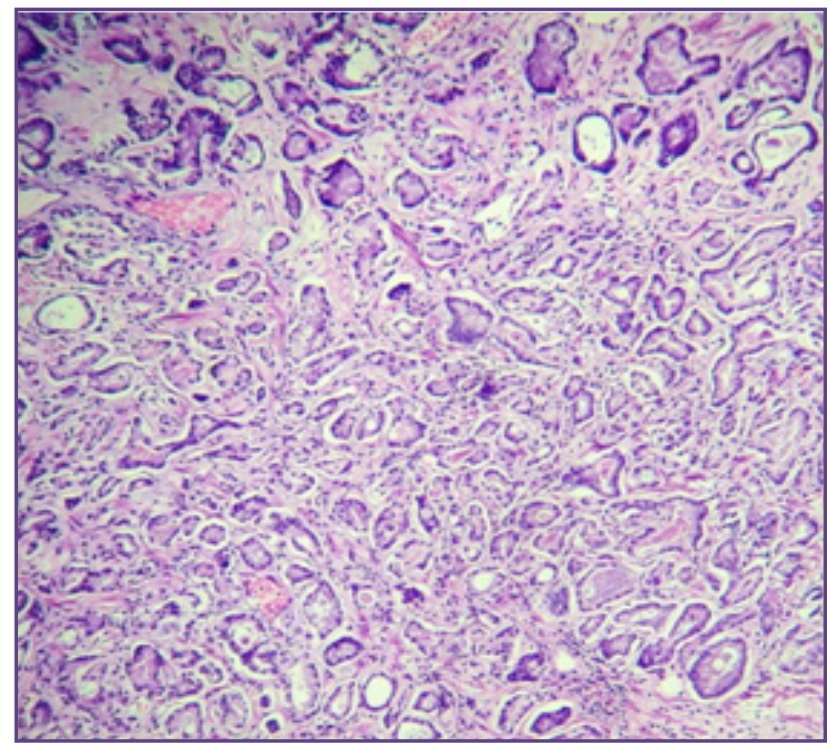

Fig. 3: Gleason grade 3- Single closely packed small glands lined by cells with hyperchromatic nuclei. (400x H\&E). metastasis. Urethral margin at prostatic apex was free of tumor.

Three months post operative, the patient did not have any complaints. The Serum PSA level was $0.1 \mathrm{ng} / \mathrm{ml}$. Currently, the patient is being followed up.

\section{Discussion}

The frequency of incidentally discovered carcinoma prostate in cystoprostatectomy specimens is extremely

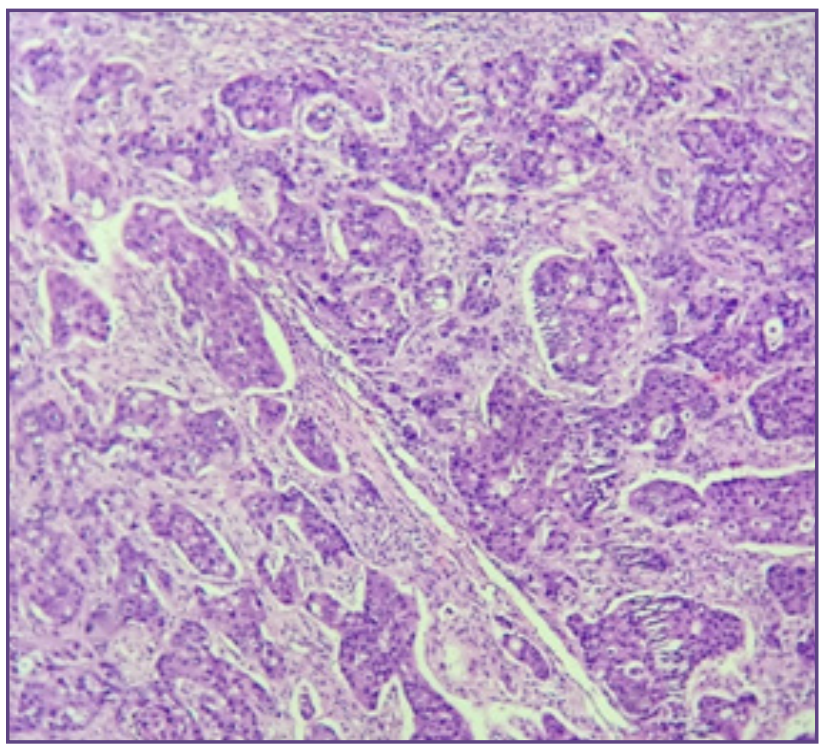

Fig. 2: High- grade urothelial carcinoma- Nests of malignant urothelial cells with nuclear pleomorphism. (100x H\&E).

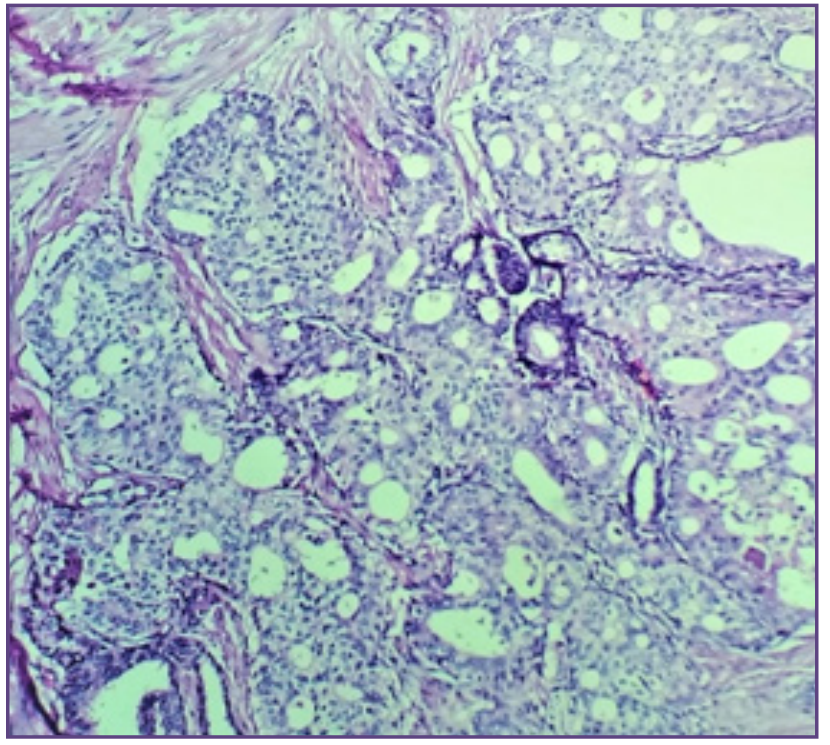

Fig. 4: Gleason Grade 4- Show crowded back to back glands. (400x H\&E). 
variable ranging from $4 \%$ to $60 \%{ }^{[1]}$ The incidence in Asia is found to be relatively lower than in Western countries. Tanaka et $\mathrm{al}^{[6]}$ from Japan have reported an incidence of $18.1 \%$ while Pan et $\mathrm{al}^{[7]}$ from China observed an incidence of $6.8 \%$. An Indian study by Mahadik et al have reported a prevalence of $12.4 \% .^{[8]}$ Out of the 22 Radical Cystoprostatectomies done for Carcinoma bladder in our institute, in the last five years, this is the first case of co existing Carcinoma Prostate. Carcinoma prostate is discovered better when the entire prostate gland is studied by taking $2-3 \mathrm{~mm}$ slices from the base to the apex. ${ }^{[1]}$

Heidegger et all in their study of 213 patients who underwent radical cystoprostatectomy for carcinoma bladder observed an incidence of $53.1 \%$ of synchronous prostate adenocarcinoma. The mean age was 71 years, Gleason score was $\geq 7$ in $86 \%$ cases and $10 \%$ had extraprostatic extensions. Both PSA and PSA\% were significantly increased in these patients. They also observed adverse bladder histology $>$ pT3 in $63.7 \%$ patients $^{[2]}$ Our patient was 82 years old and the pre operative Serum PSA was $3.4 \mathrm{ng} / \mathrm{ml}$.

Saad et al in their study of 425 cystoprostatectomy specimens done for carcinoma bladder observed prostatic adenocarcinoma in $21 \%$ and High grade prostatic intraepithelial neoplasia(HGPIN) in $10 \%$ of cases. In their review, they have described a variable incidence of $14-60 \%$ prostate cancer. They concluded that entire sampling of the prostate gland and slice thickness are critical in detecting carcinoma prostate. The PSA levels did not indicate the presence of incidental carcinoma. Also, it did not reflect the Gleason score or $\mathrm{pT}$ stage of cancer. ${ }^{[3]}$

The criteria for clinically significant cancer includes the following, ${ }^{[4]}$

- Seminal vesicle invasion

- Positive surgical margins

- $\quad$ Pathologic Gleason score $>6$

- Pelvic lymph node involvement or

- Tumor volume $>0.5 \mathrm{cc}$

Apart from the above, other criteria used by the authors were, ${ }^{[5]}$

- $\quad$ Proliferative index $>5 \%$

- $\quad$ Stage pT3

- Extracapsular extension

In our case, the Gleason score was $>7$, tumor volume was $6 \mathrm{cc}$, thus making it a clinically significant cancer. No evidence of HGPIN was seen.
Joshua et al in their study of 307 patients identified Carcinoma Prostate in $33.6 \%$ of patients. One- third of these had clinically significant cancer. ${ }^{[4]}$

Autorino et al in their review observed that the percent of clinically significant cancer varied from $5-60 \%$ in various studies. ${ }^{[5]}$

Joshua et al suggested an initial post RCP PSA within 1-2 months post operative, following annually thereafter in the absence of aggressive features like Gleason score 8-10, pelvic nodal or seminal vesicle involvement, multifocal and large extent of prostate involvement. Adjuvant hormonal or radiation therapy is not advocated. They concluded that the Ca prostate has little impact on the natural history of Carcinoma bladder. ${ }^{[4]}$ Three months post operative, Serum PSA levels in our patient was $0.1 \mathrm{ng} / \mathrm{ml}$ and he does not have any complains at present.

Androulakakis et al also observed that the simultaneous occurrence of these carcinomas does not affect the prognosis of either disease. ${ }^{[1]}$

Konski et al concluded that the most significant prognostic factor was the stage of Bladder carcinoma. ${ }^{[1]}$

\section{Conclusion}

Incidental prostatic carcinoma in patients with Carcinoma Bladder has a variable incidence. A complete sampling of the prostate will enhance the detection rates of these tumors. When prostate carcinoma is incidentally detected in radical cystoprostatectomy specimen done for Carcinoma Bladder, no additional treatment is required and these patients should be followed up as per the standard protocol. In Carcinoma Bladder patients where prostate sparing surgery is planned, routine TRUS biopsy of prostate may be done preoperatively.

\section{Acknowledgements}

Dr. Leena Naik, Prof and HOD, Dept of Pathology, Lokmanya Tilak Municipal Medical College, Mumbai

\section{References}

1. Damiano R, Lorenzo GD, Cantiello F et al. Clinicopathologic features of prostate adenocarcinoma incidentally discovered at the time of radical cystectomy: an evidence based analysis. Eur Urol 2007;52:648-57.

2. Heidegger I, Oberaigner W, Horninger W, Pichler R. High incidence of clinically significant concomitant prostate cancer in patients undergoing radical cystectomy for bladder cancer: a 10-year single center experience. Urol Oncol 2017;35:152e1-e5.

3. Saad M, Abdel-Rahim M, Abol-Enein H, Ghoneim MA. Concomitant pathology in the prostate in cystoprostatectomy 
specimens: a prospective study and review. BJU Int 2008;102:1544-50.

4. Kaelberer JB, O'Donnel MA, Mitchell DL et al. Incidental prostate cancer diagnosed at radical cystoprostatectomy for bladder cancer: disease specific outcomes and survival. Prostate Int 2016;4:107-12.

5. Autorino R, Lorenzo GD, Damiano R et al. Pathology of prostate in radical cystectomy specimens: a critical review. Surg. Oncol. 2009;18:73-84.

6. Tanaka $\mathrm{T}$, Koie $\mathrm{T}$, Ohyama $\mathrm{C}$ et al. Incidental prostate cancer in patients with muscle-invasive bladder cancer who underwent radical cystoprostatectomy. Jpn J Clin Oncol. 2017;47:1078-1082.

7. Pan J, Xue W, Sha J et al. Incidental Prostate Cancer at the Time of Cystectomy: The Incidence and Clinicopathological Features in Chinese Patients. PLoS One. 2014;9(4):e94490.

8. Mahadik R, Bijalwan P, Thomas A. Prevalence and Pathological Analysis of Adenocarcinoma Prostate Discovered Incidentally in Radical Cystoprostatectomy Specimen: An Indian Center Experience. Journal of Clinical and Diagnostic Research. 2018;12:XC01-XC03.

*Corresponding author:

Dr. Anitha Padmanabhan (Assistant Professor) Address: D-30,2/2 Sagar Sangam CHS Sector 4, Nerul, Navi Mumbai- 400706, INDIA

Phone: +91 9820458848

Email: anithahari2001@hotmail.com

Financial or other Competing Interests: None. 